\title{
The Platelet Delta Granule and Its Role in Hemostasis.
}

\author{
WT Gunning
}

Medical College of Ohio, Toledo, $\mathrm{OH} 43614$

The platelet is a dynamic hematological cell that has a half-life of approximately 5-7 days in the circulation and it is essential for hemostasis. These cells contain numerous substances that mediate both clotting and thrombolysis that are stored principally in two types of organelles, alpha and delta (dense) granules. Storage pool deficiency (SPD) is a heterogeneous bleeding disorder due to platelet dysfunction and usually related to a decreased number of these specific platelet organelles. Deficiencies of platelet alpha granules ( $\alpha$ SPD), dense granules ( $\delta$ SPD), and combined deficiencies of both alpha and delta granules and their specific substance contents will all manifest as a hemorrhagic diathesis. Patients having SPD often present with a history of easy bruising, frequent epistaxis, and a prolonged bleeding time with or without abnormal aggregation tests. Both hereditary and acquired etiologies have been reported. Chédiak-Higashi, Grey Platelet, HermanskyPudlak, and Wiskott-Aldrich syndromes are examples of hereditary SPDs. Acquired SPDs have been reported in subjects suffering from myeloproliferative disorders, chirrosis, and uremia.

Delta storage pool deficiency $(\delta$-SPD) is characterized by a decrease in platelet adenine nucleotide concentration as manifested by normal or decreased ATP, decreased ADP, and an increased ATP/ADP ratio (usually $>2.5$ ). $\delta$-SPD may be due to either a decreased number of dense granules [DG deficiency (DGD)] or the presence of a normal number of micro-DG [1]. The diagnosis of $\delta$ SPD is easily made by the platelet whole mount technique for electron microscopy (Figure 1.) [2]. The results of platelet EM correlate well with the concentration of adenine nucleotides extracted from platelets (Graphs I and II) [3]. Previous work from this laboratory has shown that DGD may be responsible for unexplained bleeding [4] as well as women with non-gynecologic menorrhagia [5]. Although the incidence of $\delta$-SPD is increased in family members of pediatric patients diagnosed with $\delta$-SPD [6], the incidence of $\delta$-SPD in the general population is unknown. Although the pattern of referral of patients for bleeding evaluation is likely to be biased, we believe that $\delta$-SPD may be more prevalent in the general population than von Willebrand disease. Thus, we have recently obtained random umbilical cord blood samples at a large Midwestern hospital in an attempt to determine the incidence of $\delta$-SPD in the general population.

We have found that the number of platelet dense granules is universally decreased in cord blood compared to normal adults (Graph I) and that the ATP and ADP contents of cord blood platelets are significantly decreased compared to those found in normal adults (Graph II \& III). The markedly decreased ATP content suggests that cord blood platelets are globally depleted of adenine nucleotides rather than dense granule deficient. Although the adenine nucleotide content is decreased globally, the ratio of ATP/ADP is similar to that of adult controls. The ATP/ADP ratio results suggest that at least 5/61 (8\%) samples evaluated are potentially $\delta$-SPD based on an ATP/ADP ratio $>4.00$. No significant correlation exists between the adenine nucleotide concentrations and dense granule number (Graph VI \& VII). Adenine nucleotide ratios tend to remain constant independent of DG number. These data suggest that neonates are born with a transient condition of depleted platelet adenine nucleotide content. This study suggests that $\delta$-SPD may be more prevalent in the general population than von Willebrand disease. 


\section{References}

1. Athota KP, et al. Blood 92:34a, 1998.

2. White JG. Seminars in Thrombosis \& Hemostasis 24:163-168, 1998.

3. McKenzie MM, et. Blood 90:140a, 1997.

4. Lachant NA, and Gunning WT Blood 98:252a, 2001.

5. Kouides P, et al. Blood 96:254a, 2000.

6. Stein DT, et al. Blood 96:255a, 2000.

A

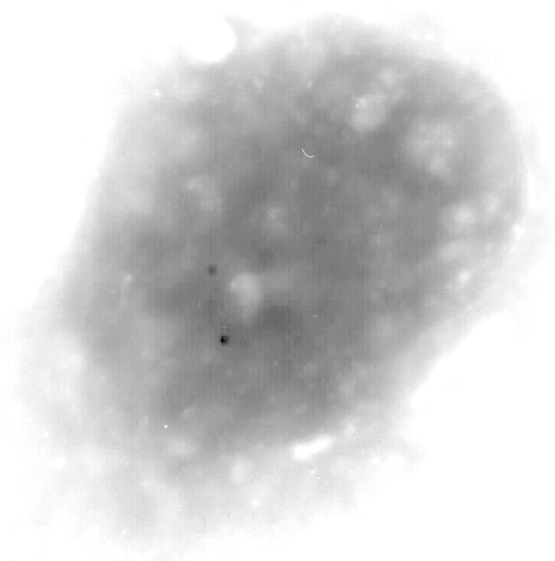

B

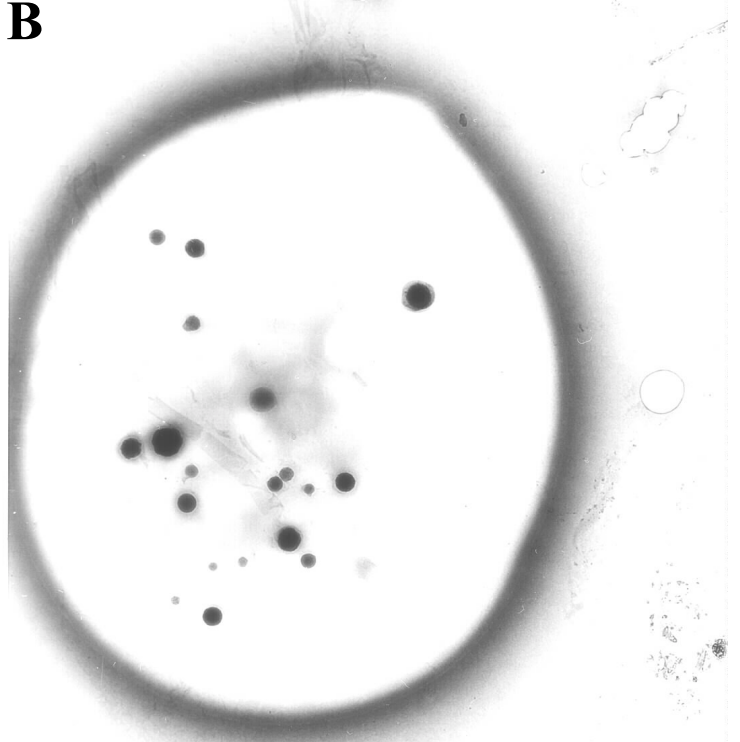

Figure 1. Electron photomicrographs of whole mounted platelets. A) Platelet with two small dense granules. B) Platelet with numerous dense granules.

Graph I. Comparison of Mean Dense Granule Number in Platelets Obtained from Cord Blood and Adult Peripheral Blood

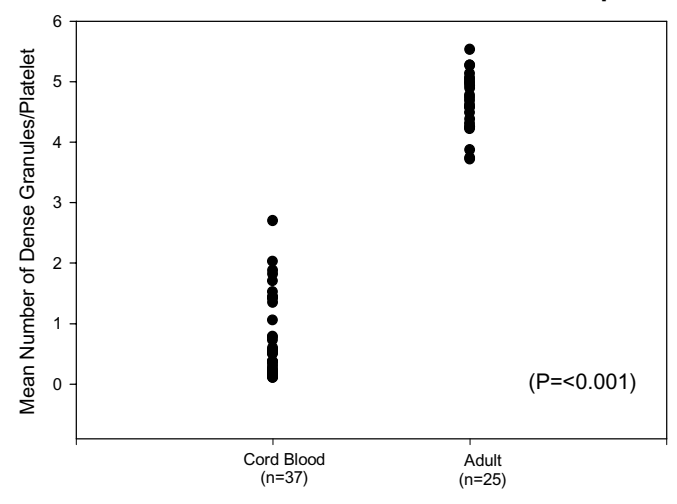

Graph II. Comparison of Dense Granule Number and Adenine Nucleotide Content in Cord Blood and Adult Platelets

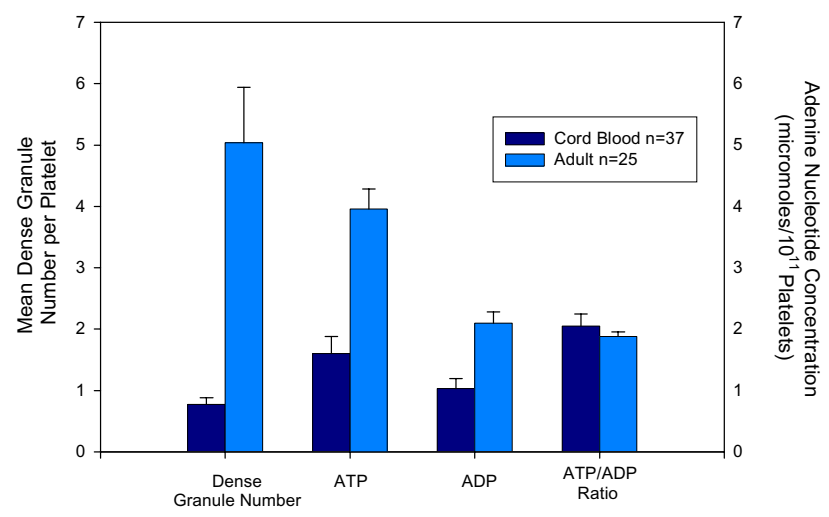

